\title{
On-Tissue Protein Identification and Imaging by MALDI-Ion Mobility Mass Spectrometry
}

\author{
Jonathan Stauber, ${ }^{\mathrm{a}}$ Luke MacAleese, ${ }^{\mathrm{a}, \mathrm{d}}$ Julien Franck, ${ }^{\mathrm{b}}$ \\ Emmanuelle Claude, ${ }^{\mathrm{c}}$ Marten Snel, ${ }^{\mathrm{c}}$ Basak Kükrer Kaletas, ${ }^{\mathrm{a}}$ \\ Ingrid M. V. D. Wiel, ${ }^{\mathrm{a}}$ Maxence Wisztorski, ${ }^{\mathrm{b}}$ Isabelle Fournier, ${ }^{\mathrm{b}}$ \\ and Ron M. A. Heeren ${ }^{\mathrm{a}, \mathrm{d}}$ \\ ${ }^{a}$ FOM Institute for Atomic and Molecular Physics, Amsterdam, The Netherlands \\ ${ }^{\mathrm{b}}$ MALDI Imaging Team, University of Lille, Lille, France \\ ${ }^{c}$ Waters Corporation, Manchester, United Kingdom \\ d The Netherlands Proteomics Centre, Utrecht, The Netherlands
}

MALDI imaging mass spectrometry (MALDI-IMS) has become a powerful tool for the detection and localization of drugs, proteins, and lipids on-tissue. Nevertheless, this approach can only perform identification of low mass molecules as lipids, pharmaceuticals, and peptides. In this article, a combination of approaches for the detection and imaging of proteins and their identification directly on-tissue is described after tryptic digestion. Enzymatic digestion protocols for different kinds of tissues-formalin fixed paraffin embedded (FFPE) and frozen tissues-are combined with MALDI-ion mobility mass spectrometry (IM-MS). This combination enables localization and identification of proteins via their related digested peptides. In a number of cases, ion mobility separates isobaric ions that cannot be identified by conventional MALDI time-of-flight (TOF) mass spectrometry. The amount of detected peaks per measurement increases (versus conventional MALDI-TOF), which enables mass and time selected ion images and the identification of separated ions. These experiments demonstrate the feasibility of direct proteins identification by ion-mobility-TOF IMS from tissue. The tissue digestion combined with MALDI-IM-TOF-IMS approach allows a proteomics "bottom-up" strategy with different kinds of tissue samples, especially FFPE tissues conserved for a long time in hospital sample banks. The combination of IM with IMS marks the development of IMS approaches as real proteomic tools, which brings new perspectives to biological studies. (J Am Soc Mass Spectrom 2010, 21, 338-347) (c) 2010 American Society for Mass Spectrometry

I maging mass spectrometry (IMS) is a mass spectrometry technique applied to chemical analysis of surfaces. Ion beams for secondary ion mass spectrometry (SIMS) [1-4] and lasers for matrix-assisted-laserdesorption-ionization mass spectrometry (MALDI-MS) [5-8] are commonly used surface probes for IMS, which typically provide nanometer and micrometer lateral resolution, respectively. The application of IMS to the analysis of biological surfaces and tissues has emerged more recently [9-13]. The intrinsic label-free capabilities of IMS allow the spatial distribution of each molecule on the surface to be measured simultaneously by the detection of its specific molecular weight. The chemical and localization information obtained by IMS can be compared with the results of immuno-histochemistry (IHC) [14]. However, IMS is also a discovery-oriented tool for nontargeted investigation of thousands of molecules in a single experiment [15]. IMS integrates naturally into proteomics workflows for peptide and protein

Address reprint requests to Professor R. M. A. Heeren, FOM Institute for Atomic and Molecular Physics, Macromolecular Ion Physics, Biomolecular Mass Spectrometry, Science Park 104, 1098 XG Amsterdam, The Netherlands. E-mail: heeren@amolf.nl analysis and identification on-tissue, which has led to a number of recent biological applications [16].

However, the potential of IMS integration into proteomics field is still compromised by two issues: the sensitivity of detection and the identification capabilities of low abundant peptides are rather low compared with other classic proteomics techniques. Between 1000 and 3000 molecules are usually detected and correspond to the most abundant ionizable peptides. The biological applications of IMS for peptides and proteins mainly use MALDI-MS. The lateral resolution is limited by the laser spot size rastering the surface (in microprobe mode). High-resolution MALDI-MS instruments, such as stigmatic ion microscopes [17, 18], circumvent this issue but the detection of full mass spectra can be problematic, in most cases due to slow detectors (CCD cameras, phosphor screens, or delay lines) [19]. The majority of IMS experiments are performed in the microprobe MALDI-SIMS mode, where high spatial resolution is limited by the loss of sensitivity and chemical damage due to highly focused laser beams [20].

Therefore, one of the main concerns for IMS is on-tissue detection sensitivity. Low sensitivity is caused 
by several factors such as the low concentration of each analyte and the large chemical complexity of microenvironments, which is responsible for ion suppression effects. Sample preparation methods play a crucial role in enhancing sensitivity on-tissue [14, 21]. In addition, instrumental sensitivity of TOF mass spectrometers usually decreases at high $m / z$, which are currently the most widespread instruments in use for IMS. These limitations slow the development of on-tissue identification, which is needed to make IMS a practical tool for proteomics.

Sample preparation protocols, which include tissue washes and careful matrix deposition, are used to minimize ion suppression effects and enhance availability of analytes at the surface. Specific protocols were developed for both fresh-frozen and fixed tissues (typically formalin-fixation followed by paraffin embedding FFPE) [22-24]. The analysis of FFPE tissue opens hospital sample banks, which represent an enormous amount of well documented tissues, whereas fresh samples are sometimes more scarce. Other sample preparation and instrumental strategies include, e.g., targeting chemical families with functionalized nanoparticles and development of post-ionization sources [25-28].

A powerful complementary technique to direct protein analysis is on-tissue enzymatic digestion [22, 2931]. Protein digestion typically yields peptides between 400 and $3500 \mathrm{Da}$, a range where instrumental sensitivity is higher than that for intact proteins. However, the mass spectrum is more complicated in the low mass region. In addition, enzymatic digestion enables efficient tandem MS on small single charged peptides in TOF-based instruments. Current approaches for identification of digested proteins or peptides from tissue involve extraction and separate tandem-MS measurements, sometimes followed by comparison with ontissue peptide mass fingerprinting (PMF) [29]. Tissue chemical complexity prohibits efficient PMF. Further, the mass resolving power and mass accuracy of TOF instruments are usually insufficient for exact mass detection of all measured intact proteins, though FT-MS methods are available [32]. MS/MS has scarcely been performed directly on-tissue; typically on the most abundant peptides [30] or for disambiguation between isobaric compounds rather than absolute identification $[33,34]$. However, the chemical complexity on-tissue may result in multiple peptide ions at each nominal mass. As a consequence, except for higher abundant peptides, identification by MS/MS may prove difficult due to fragments from different peptides.

Here we describe the combination of ion mobility (IM) and ion mobility mass spectrometry (IMMS) to separate the different peptide contributions to MS/MS spectra from a single mass-selected peak [35-40]. IM adds a separation dimension (reported as drift time) to mass spectrometry, which is comparable to the retention time of liquid chromatography (LC) and gas chromatography (GC) hyphenated MS methods. Since its inception in 1980s, ion mobility has evolved rapidly, with the emergence of alternatives to the classic drift tubes: field asymmetric waveform ion mobility spectrometry (FAIMS) and, these days, the traveling wave (T-wave) [41, 42]. Initially, IM was used to differentiate conformers in the gas phase, but more recently IMMS has been applied to protein conformer differentiation, top-down protein sequencing, noncovalent protein complexes, isobaric compound identification, and imaging by mass spectrometry [43-46]. IM benefits IMS in two major ways. First, the addition of a separation dimension enables the detection of more MS peaks. IM decongests the mass spectrum and allows the identification of trends and chemical families, although, IM does not enhance sensitivity since it does not modify the ionization conditions. Secondly, the combination of mass with drift time selection criteria enables the differentiation of isobaric peptide contributions to a fragmentation spectrum. In this article, we present the high sensitivity identification of multiple digested peptides on-tissue with use of a MALDI-IM-IMS instrument (SYNAPT-HDMS; Waters Corp., Manchester, UK), and we show that IM-IMS enables the identification with high sensitivity of numerous peptides on-tissue which correspond to the additional step that was missing to the actual incorporation of IMS into proteomic workflows.

\section{Experimental}

\section{Materials and Methods}

$\alpha$-Cyano-4-hydroxycinnamic acid (HCCA), 2,5 dihydroxibenzoic acid (2,5-DHB), ammonium bicarbonate, trisma base, toluene, ethanol, angiotensin II, Des-Argbradykinin, Substance P, ACTH 18-39, ACTH 7-38 (Adreno CorticoTropic Hormone), trifluoroacetic acid (TFA)n and bovine insulin were obtained from SigmaAldrich, (Zwijndrecht, The Netherlands) and used as provided. Trypsin was purchased from Promega (Madison, WI, USA) and acetonitrile (ACN) and methanol from Biosolve (Westford, MA, USA).

\section{Animal Surgery and Tissue Preparation}

Wistar male rats (180-200 g) were anesthetized with pentobarbital (50 mg/kg, i.p.) and sacrificed. Their brains were removed and fixed for $40 \mathrm{~min}$ at $48^{\circ} \mathrm{C}$ in $4 \%$ paraformaldehyde. Brain tissue blocks were stored in a box at room temperature for $12 \mathrm{y}$ before the experiments described herein. Frozen human cerebellum was provided by EMC Rotterdam (Erasmus Medical Center). It was fast-frozen with cold isopropane $\left(-50^{\circ} \mathrm{C}\right)$ and directly conserved in a $-80^{\circ} \mathrm{C}$ freezer for 1 mo before use.

\section{Tissue Dewaxing and Treatment}

Tissue sections of $10 \mu \mathrm{m}$ were obtained by microtome (Microm HM 525; Walldorf, Germany) and applied to 
indium tin oxide (ITO) coated conductive glass slides. Paraffin was removed by two baths of $5 \mathrm{~min}$ in toluene and lightly rehydrated with graded ethanol $\left(100^{\circ}, 96^{\circ}\right.$, $70^{\circ}$, and $30^{\circ}$ ) baths before drying at room temperature. Frozen tissues were immerged in chloroform for $5 \mathrm{~s}$ before use.

\section{In Situ Trypsin Digestion and Matrix Deposition}

Trypsin microspotting: spots of enzyme (trypsin at 0,05 $\mu \mathrm{g} / \mu \mathrm{L}$ in water) were deposited by a high-accuracy position automatic chemical inkjet printer (CHIP-1000; Shimadzu Biotech, Kyoto, Japan). The whole tissue section was microspotted with enzyme in a $150 \mu \mathrm{m}$ spacing raster scheme. After optimization, the quantity of trypsin was set to $20 \mathrm{~nL}$ deposited in 40 cycles of 500 $\mathrm{pL}$ at each spot position to cover the entire tissue surface. HCCA matrix was deposited by a vibrational sprayer system (ImagePrep; Bruker Daltonik $\mathrm{GmbH}$, Bremen, Germany). The matrix solution was HCCA (10 $\mathrm{mg} / \mathrm{mL})$ in ACN/TFA $0.1 \%,(6: 4, \mathrm{vol} / \mathrm{vol})$ and was sonicated for $2 \mathrm{~min}$.

\section{Mass Spectrometry}

\section{MALDI MS and MSI Tissue Sections After In Situ Automatic Trypsin Digestion}

FFPE and frozen tissue section analysis after in situ digestion was performed in an Ultraflex III TOF-TOF instrument (Bruker Daltonik GmbH, Bremen, Germany) and a Synapt MALDI-Q-ion mobility TOF (Waters Corp.). The Ultraflex is equipped with a LIFT III cell and Smartbeam laser with a repetition rate up to 200 Hz. The Synapt is equipped with a YAG laser with a repetition rate of $200 \mathrm{~Hz}$ and a T-wave ion mobility cell. The ion mobility separation parameters were: trap gas flow $1.5 \mathrm{~mL} / \mathrm{min}$ (argon), IMS gas flow $22 \mathrm{~mL} / \mathrm{min}$ (nitrogen), and ion mobility wave velocity $300 \mathrm{~m} / \mathrm{s}$. (a)

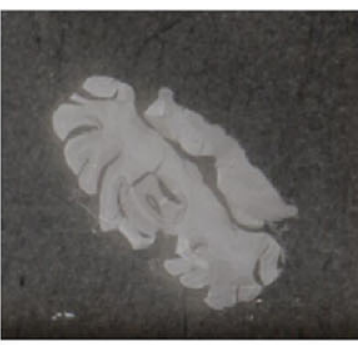

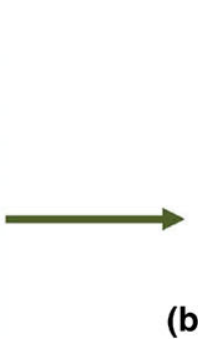

(b)

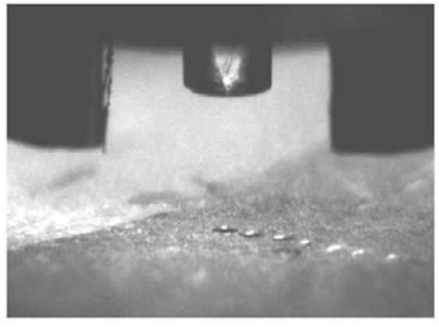

Microprobe Mode (c)

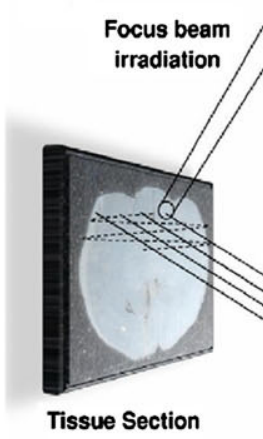

(d)

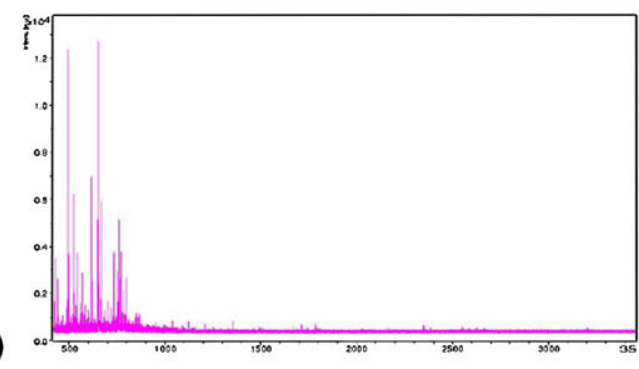

(e)

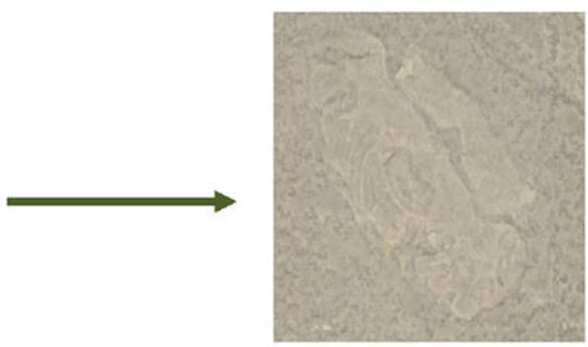

Figure 1. Workflow for FFPE or frozen on-tissue protein identification. The image shows the used steps of matrix deposition (b) on rat brain tissue (a). After MALDI imaging experiment (c), mass spectra of undigested (d) or digested (e) tissue are obtained. Then images can be reconstructed (c). 
External calibration was performed using a solution of standard peptides (bradykinin $1.6 \mu \mathrm{M}$, Substance P 1.6 $\mu \mathrm{M}, \mathrm{ACTH} 18-391.6 \mu \mathrm{M}, \mathrm{ACTH}$ 7-38 $3.2 \mu \mathrm{M}$, bovine insulin $4.8 \mu \mathrm{M}$, and bovine ubiquitin $4.8 \mu \mathrm{M}$ in $\mathrm{H}_{2} \mathrm{O}$ ) and a polyethylene glycol (PPG-2000). The spatial resolution (determined by the laser spot size) of the Bruker Ultraflex instrument was $75 \mu \mathrm{m}$, while the Synapt spatial resolution was $150 \mu \mathrm{m}$. FlexImaging ver. 2.0 software (Bruker Daltonik GmbH, Bremen, Germany) and Biomap (Novartis, Basel, Switzerland) were used for image reconstruction. Around 13,000 irradiated po- sitions are recorded for a full brain tissue with 800 laser shots per position. The software (Biomap and FlexImaging) measures an average mass spectrum at each position on the tissue section.

\section{MALDI MS/MS with Ion Mobility}

MALDI MS/MS was performed with the Synapt MALDI ion mobility-TOF with the same ion mobility parameters as described in the previous section, performed in the last part of the T-wave called the "transfer
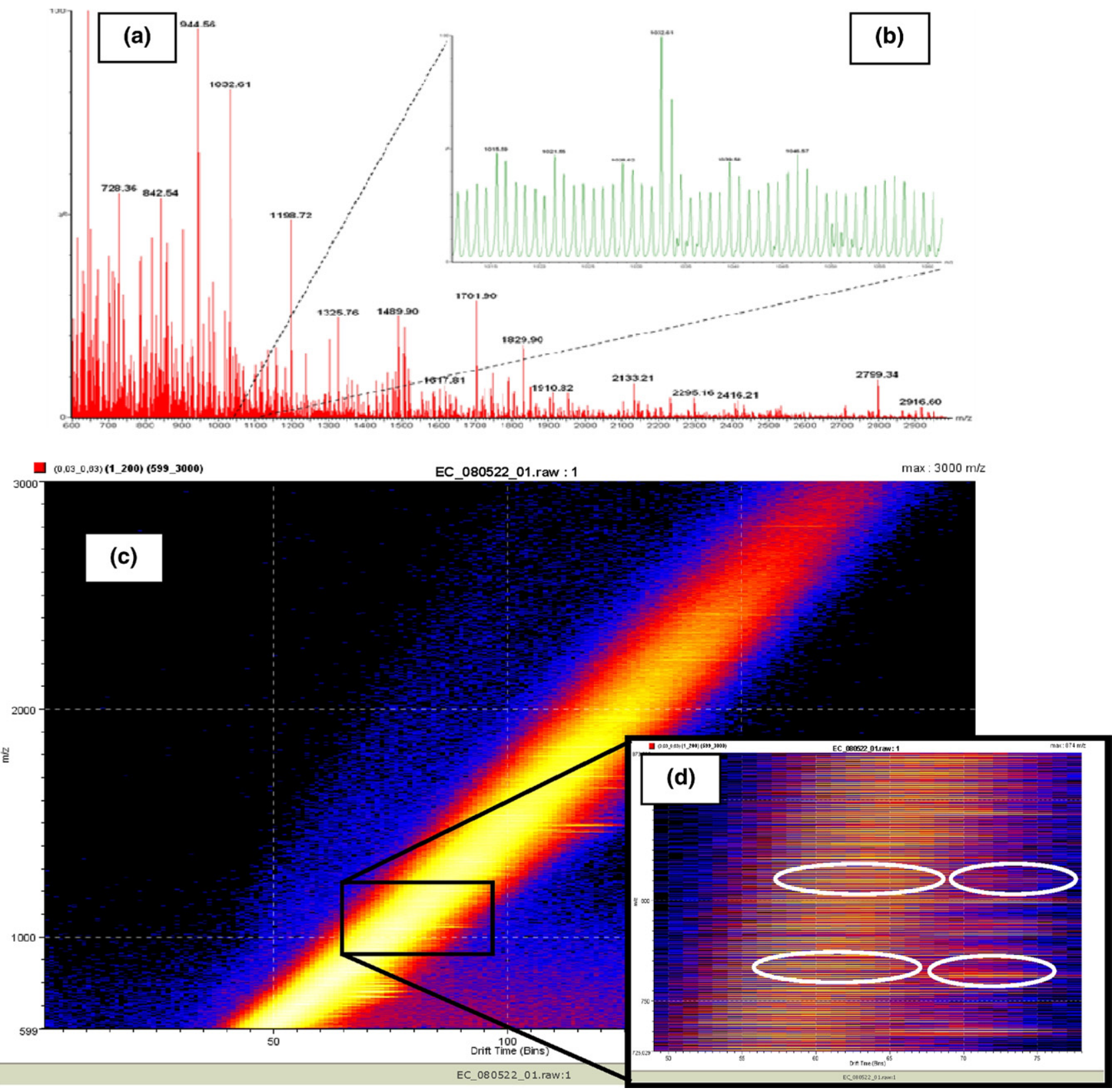

Figure 2. Mass spectra of digested FFPE tissue (a) and zoomed around $1040 \mathrm{~m} / \mathrm{z}$ (b) and the corresponding drift scope from MALDI-Ion mobility mass spectrometry (c). A zoomed area in the driftscope (d) shows isobaric ions with different drift times in the circled areas. The driftscope corresponds to the 3-dimension visualization of the $m / z$ value, signal intensities, and the retention time inside the ion mobility cell. 
cell". The collision energy in the transfer cell was between 75 and $85 \mathrm{eV}$. Protein identification was performed with MASCOT sequence query search program using the SwissProt database restricted to the taxonomy "Rattus norvegicus" for FFPE tissue and "Homo sapiens" for frozen tissue. A tolerance of $20 \mathrm{ppm}$ for peptide and 0.1 Da for MS/MS was set.

\section{Results and Discussion}

\section{Sample Preparation}

Figure 1 presents the IMS workflow used herein. Sample sections were submitted to different washing steps, as described in the Experimental section, to avoid formation of salt adducts and in general to reduce ion suppression effects. In the case of FFPE tissues, samples were dewaxed [47] with baths of xylene or toluene and a dilution series of ethanol bathes. The dewaxing and hydration steps are very crucial to have a hydrophilic sample, which can easily incorporate trypsin solution. For frozen samples, chloroform was used to remove lipids and consequently facilitate MS/MS identifications of low mass peptides. A trypsin digestion protocol was then applied to FFPE and frozen tissue sections for the identification of peptides and proteins in tissue. Trypsin was spotted on-tissue and digestion was fol-

(a)
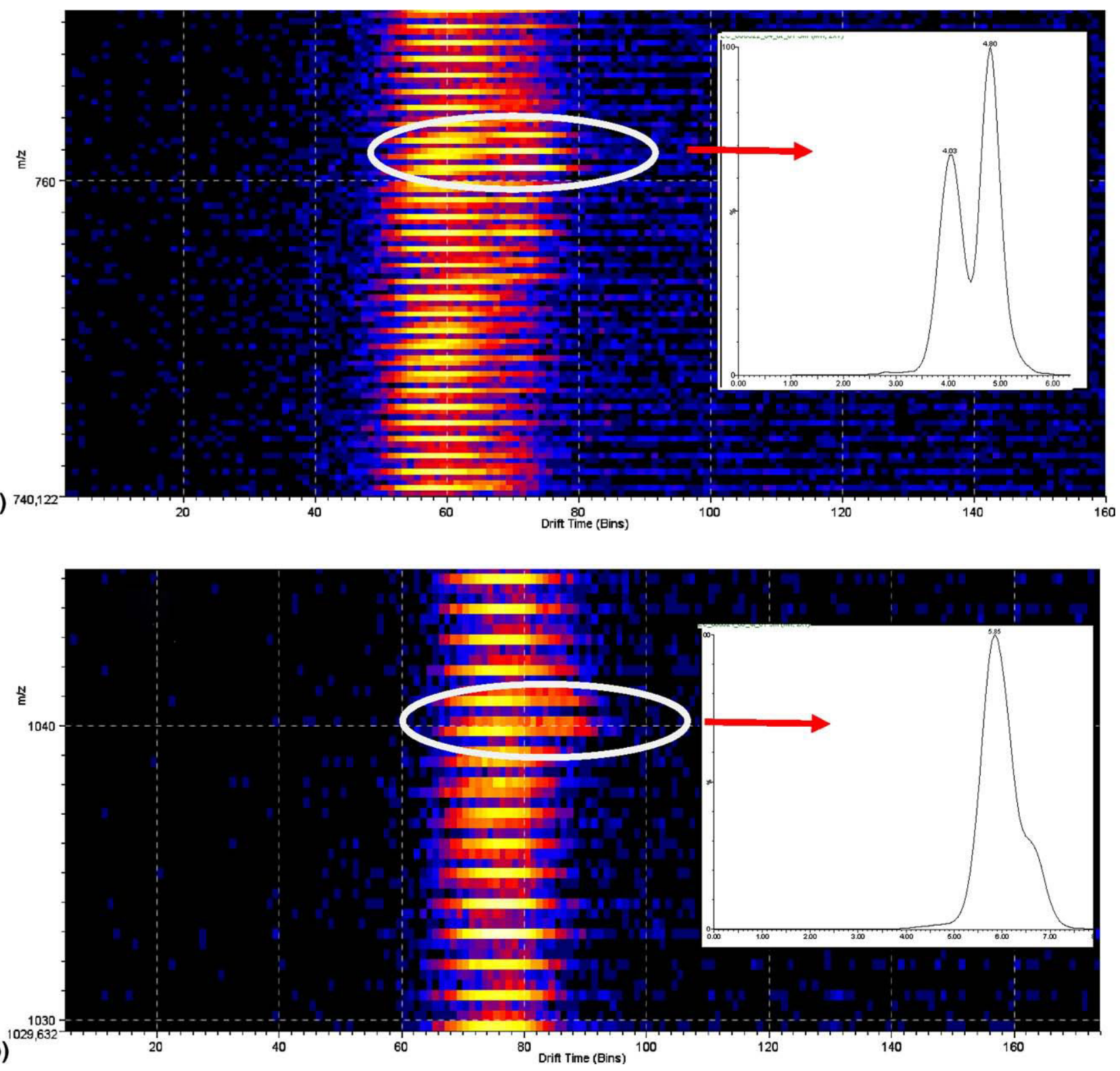

Figure 3. These images show the ability to separate digested peptides by ion mobility mass spectrometry. Two examples show separations of ions by ion mobility according their drift time (a). Two isobaric ions of $760 \mathrm{~m} / \mathrm{z}$ from frozen cerebellum tissue (framed) (b). Two isobaric ions of $1039 \mathrm{~m} / \mathrm{z}$ from FFPE cerebellum tissue (framed). Even if the resolution is low, isobaric ions are separated and identified by selecting their specific drift time (image 5). 
lowed by a cold ethanol wash by immersion to eliminate salts from the trypsin buffer solution. Afterwards, matrix was deposited on the tissue. The digestion process was performed so the spatial distribution of compounds on the digested tissue surface would be retained. The homogeneity of both trypsin and matrix layers is ensured by reproducible mechanical deposition.

The comparison between digested tissue and control tissue (Figure 1d and e) shows large differences in terms of number of detected peaks in the peptide mass range. The sample preparation seems to be a crucial step for detection sensitivity and efficient reproducibility of analysis. In this experiment, trypsin and matrix were spotted on top of sample surface. These steps increase the risk of delocalization of peptides. Therefore, reproducible and robust techniques are needed to overcome these delocalization problems.

\section{Digested Sample Analysis}

On-tissue digestion results in a large number of peaks as observed in Figure 2a, especially in the mass range $400-2500 \mathrm{~m} / \mathrm{z}$. Figure $2 \mathrm{~b}$ shows a zoom inset where a large number of peaks are observed in a small mass range (1-2 Da). The large number of peptides results in an elevated baseline level and peaks without any isotopic profile. This large number of peptides cannot be discriminated and selected individually for MS/MS identification by classic mass selection criterion in, e.g., MALDI-TOF/TOF instruments. Two solutions for complicated mass spectra, such as those shown in Figure 2, can be envisaged. A high resolution mass spectrometer such as FT-MS can be used or a gas-phase ion mobility separation before mass analysis can be added.

MALDI-ion mobility MS brings an added value to MALDI-MS tissue imaging by the separation of different compounds families such as lipids and proteins [44, 46]. The ion mobility cell, positioned between a quad- rupole and a time-of-flight analyzer, allows the separation of isobaric compounds or compounds with similar $m / z$, which cannot be separated by single mass criterion. Different ion conformations have different ion crosssections and result in different drift times. This particular property is comparable to liquid chromatography separation and allows the separation and identification of chemical families such as matrix, drugs, lipids, and single or double charged peptide ions by their retention time inside the ion mobility cell. As observed in the average mass spectra of a FFPE brain section measured with MALDIIM-MS in Figure 2a, many compounds have similar $m / z$, but it is possible to discriminate between them by their drift time (Figure 2c and d). Figure 2c shows the driftscope corresponding to the $3 \mathrm{D}$ visualization of detected signals. The mass scale $(\mathrm{m} / \mathrm{z})$ is the $\mathbf{y}$ axis, the drift time is the $\mathbf{x}$ axis, and the color scale representing the signal intensity constitutes the third dimension of the driftscope. Zooming on the driftscope shows isobaric ions with different drift times (circled, Figure 2d). The added drift time dimension (a rapid separation in the gas phase) is used to differentiate isobaric peptides from different proteins, for imaging MS experiments, and to identify them by tandem MS with high confidence using a combination of $\mathrm{m} / \mathrm{z}$ and drift time selection criteria.

\section{Drift Time Separation and MS Experiment in Digested Tissue}

In MS mode, without any selection in the quadrupole, ions are detected as observed in the mass spectrum (Figure 2a) and in the corresponding drift scope (Figure 2c). Many peaks are observed with different drift times but an identical nominal mass over charge ratio. Figure $3 a$ and $b$ illustrate the drift time separation of several isobaric $m / z$ peaks. The time-resolved drift time separa-
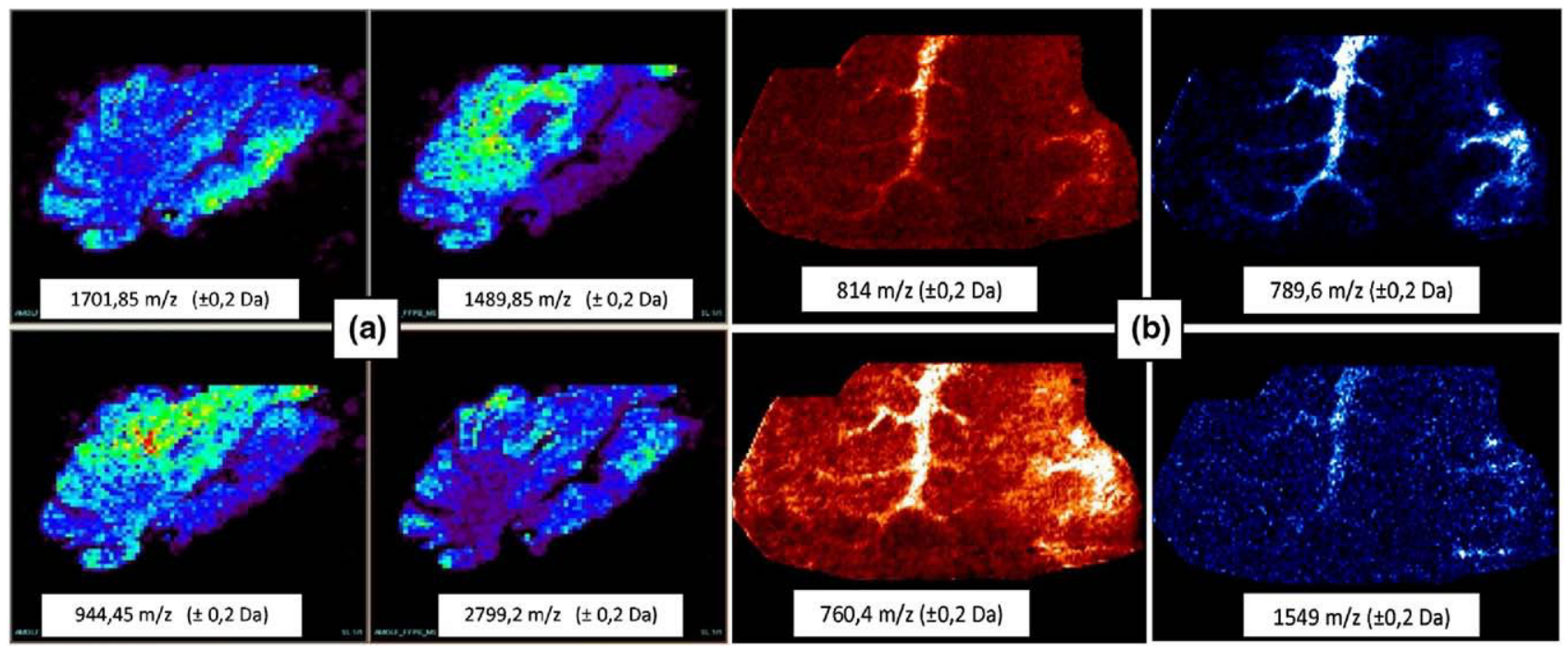

Figure 4. MALDI Imaging of different compounds in FFPE (a) and frozen (b) with ion mobility separation (Biomap software). 
tion allows the selection of different isobaric ions when reconstructing images, which allows mass and drift time selected ion images as shown in Figure 4. Isobaric ions can be distributed differently on-tissue, but mass selected ion images would display the overlaid image with contributions from every isobaric ion. Time separation enables the deconvolution of this image and reveals single compound-specific localization on-tissue. This example illustrates how ion mobility differentiates a large number of isobaric molecules by their IM retention time. MALDI-IM-IMS proves capable to detect and localize many more compounds than a classic MALDI-IMS experiment. Approximately 25\% more peaks (many of which are peptides) have been detected with the addition of ion mobility separation. IM greatly enhances the detection sensitivity in MALDI-MS and increases the biological application potentials of IMS.

\section{Proteins MS/MS Identification of Peptides Fragments}

Figure 5 shows two examples of MS/MS analysis of mass and time selected parent ions. Both parent ions mass-selected at $m / z 760$ and 1039 ions present two drift time maxima corresponding to at least two different isobaric ions. Collision induced dissociation (CID) is performed in the transfer cell in the last part of the tri-wave mobility cell. Isobaric ions are first separated then fragmented inside the transfer cell followed by mass analysis in the orthogonal-TOF. As a consequence, the drift time separation is conserved after dissociation and all fragment ions with similar retention time originate from the same parent. The ion selection window is kept relatively large $(1 \mathrm{Da})$ to maintain enough sensitivity. The $m / z 760$ isobaric ions are presented in Figure 5 together with the associated MS/MS drift scope. Two different isobaric ions and their fragments were de-

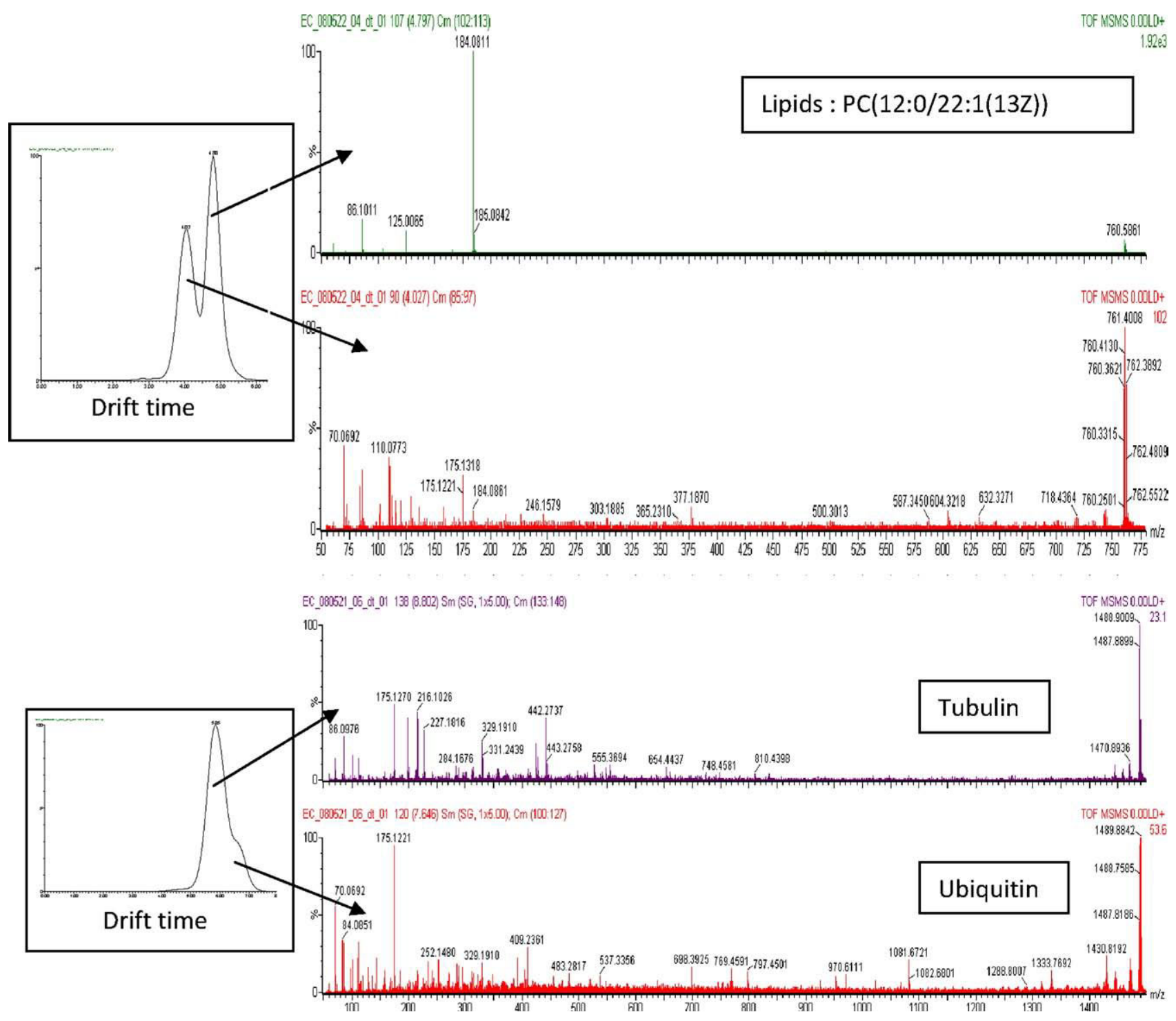

Figure 5. A. Mass spectra of fragmented peptides by MALDI-Ion mobility MS/MS. Two examples of separation of MS/MS ions are presented $(760$ and $1039 \mathrm{~m} / \mathrm{z})$. 
tected. The software can reconstruct the proper fragments when the drift-time criterion is selected. Without the ion mobility separation, the tandem MS analysis could be misleading and the ion identified as a lipid (data not shown). However, the fragmentation pattern of isobaric ions ( $m / z 760)$ appears different when separated by ion mobility (Figure 5). The characteristic observed fragment ions with $\mathrm{m} / \mathrm{z} 184$ and 175 demonstrate simultaneous presence of a lipid and a peptide. In a classic tandem MS scheme, the intensity difference between these two compounds would not allow the conclusive determination of the presence of the peptide. The second example in the bottom half of figure 5 illustrates the possibility to separate and identify two compounds within the same chemical family, where two ipeptides show unique fragmentation patterns after ion mobility separation. The spatial distribution of these ion mobility separated and fragmented peptides are shown in the images in Figure 6.

The MALDI-IM-IMS geometry allows the a posteriori separation with the drift time, where the fragments come from different parent ions. Each isobaric ion that can be observed and separated in IM-MS mode can be subjected to specific tandem-MS analysis and give its own proper fragment ion spectrum.

\section{Mascot Score}

The ability to separate isobaric ions with IM and, therefore, to separate fragmentation profiles of different ions enables a high specificity database search by pooling fragments associated to only one parent. The comparison between Mascot scores with and without drift time fragment pooling shows the power of the added IMS separation (data not shown). The score increases dramatically by a factor of five to six when ion mobilityspecific fragmentation profiles are taken into account for database search. Thus, fragmentation profiles originating from ions with $m / z 1039$ separated by ion mobility are submitted to separate Mascot database searches and lead to identification of two different peptides from two different proteins: tubulin and ubiquitin. Tubulin protein is identified with a high score whereas ubiquitin gets a lower score. However, without ion mobility separation, the Mascot score is too low and insignificant and does not allow the identification of these proteins. The ion mobility separation combined with MALDI MS/MS instrument increases the number of identified peptides by simplifying and eliminating isobaric fragments from different compounds. The ion mobility also separates lipids and peptides fragments, allowing the easy identification of phosphocholine PC $[12$ : $0 / 22: 1(13 Z)$ ]. The separation of isobaric fragments is the second added value of ion mobility separation. The combination of the two added values of ion mobility, characterized by the differential identification and differential imaging of isobaric ions, brings MALDI imaging MS a step further in the field of proteomics, enabling imaging of identified proteins and lipids.

\section{Conclusion}

The addition of IM to the MALDI-MS experiment adds a powerful separation dimension, which provides more insight into an MS dataset. In this article, this is illustrated with mass and time selected ion images representing specific tissue distributions for different isobaric tryptic peptides. However, this ability to separate isobaric ions can also be applied to peptide identification. Drift time separation of isobaric parent ions enables observation of specific fragmentation patterns, which results in higher databases search scores and higher confidence of peptides and proteins identification. This is especially useful for the analysis of digested tissues where spectra are very complex in the peptide mass range. This results in numerous peptides isotopic pattern overlaps and multiple unresolved peaks at a single nominal mass. IM coupled to tandem MS within an imaging mass spectrometry context results in the determination of accurate and relevant spatial distributions

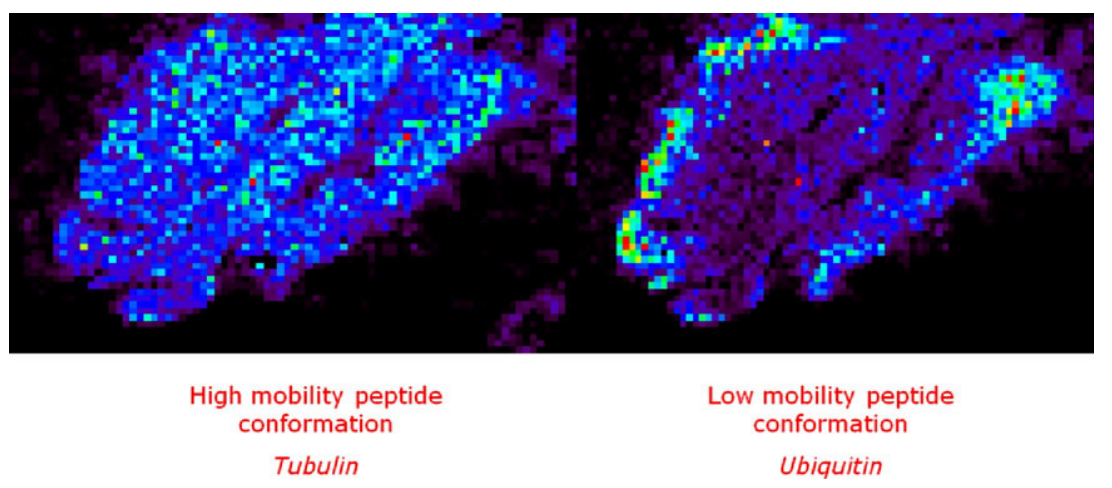

Figure 6. Images of separated and identified isobaric ions of Tubulin and ubiquitin peptide fragments $(1039 \mathrm{~m} / \mathrm{z})$ are presented directly on-tissue by ion mobility mass spectrometry imaging (Biomap software). For these images, two different drift times have been selected: 100-127 and 133-148 bins to reconstruct the respective images. Without any ion mobility, one image is obtained corresponding to the superposition of these two images. 
of identified proteins based on isobaric digested peptides. Different proteins identified, based on tandem MS of isobaric ions, display different tissue localization. This proves that IMS enhances the MALDI molecular imaging capabilities, especially by performing some peptides identification directly on-tissue. Therefore, this approach could become, in a near future, a tool for bottom-up proteomics.

\section{Acknowledgments}

The authors acknowledge that this work is part of the research program of the "Stichting voor Fundamenteel Onderzoek der Materie (FOM), which is financially supported by the Nederlandse organisatie voor Wetenschappelijk Onderzoek (NWO). The authors also acknowledge funding and collaborations through the Biomarker Discovery research program of the Netherlands Proteomics Center (NPC). We also gratefully acknowledge financial support from Meditrans, An Integrated Project funded by the European Commission under the "nanotechnologies and nanosciences, knowledge-based multifunctional materials and new production processes and devices" (NMP) thematic priority of the Sixth Framework Programme. Contract Number: NMP4-CT-2006026668 .

\section{References}

1. Jones, E. A.; Lockyer, N. P.; Vickerman, J. C. Mass Spectral Analysis and Imaging of Tissue by TOF-SIMS-The Role of Buckminsterfullerene, C-60(+), Primary Ions. Int. J. Mass Spectrom. 2007, 260, 146-157.

2. Wucher, A. Molecular Secondary Ion Formation Under Cluster Bombardment: A Fundamental Review. Appl. Surf. Sci. 2006, 252, 6482-6489.

3. Winograd, N. The Magic of Cluster SIMS. Anal. Chem. 2005, 77, 142A-149A.

4. Wong, S. C. C.; Hill, R.; Blenkinsopp, P.; Lockyer, N. P.; Weibel, D. E.; Vickerman, J. C. Development of a C-60(+) Ion Gun for Static SIMS and Chemical Imaging. Appl. Surf. Sci. 2003, 203, 219-222.

5. Karas, M.; Bachmann, D.; Bahr, U.; Hillenkamp, F. Matrix-Assisted Ultraviolet Laser Desorption of Nonvolatile Compounds. Int. J. Mass Spectrom. Ion Processes 1987, 78, 53-68.

6. Hillenkamp, F.; Karas, M. Matrix-Assisted Laser Desorption/Ionization, an Experience. Int. J. Mass Spectrom. 2000, 200, 71-77.

7. Karas, M.; Hillenkamp, F. Laser Desorption Ionization of Proteins with Molecular Masses Exceeding 10,000 Daltons. Anal. Chem. 1988, 60, 2299_ 2301.

8. Zenobi, R.; Knochenmuss, R. Ion Formation in MALDI Mass Spectrometry. Mass Spectrom. Rev. 1998, 17, 337-366.

9. Stoeckli, M.; Chaurand, P.; Hallahan, D. E.; Caprioli, R. M. Imaging Mass Spectrometry: A New Technology for the Analysis of Protein Expression in Mammalian Tissues. Nat. Med. 2001, 7, 493-496.

10. Stoeckli, M.; Farmer, T. B.; Caprioli, R. M. Automated Mass Spectrometry Imaging with a Matrix-Assisted Laser Desorption Ionization Timeof-Flight Instrument. J. Am. Soc. Mass Spectrom. 1999, 10, 67-71.

11. McDonnell, L. A.; Heeren, R. M. A. Imaging Mass Spectrometry. Mass Spectrom. Rev. 2007, 26, 606-643.

12. Garden, R. W.; Sweedler, J. V. Heterogeneity Within MALDI Samples as Revealed by Mass Spectrometric Imaging. Anal. Chem. 2000, 72, 30-36.

13. Troendle, F. J.; Reddick, C. D.; Yost, R. A. Detection of Pharmaceutical Compounds in Tissue by Matrix-Assisted Laser Desorption/Ionization and Laser Desorption/Chemical Ionization Tandem Mass Spectrometry with a Quadrupole Ion Trap. J. Am. Soc. Mass Spectrom. 1999, 10, 1315-1321.

14. Lemaire, R.; Wisztorski, M.; Desmons, A.; Tabet, J. C.; Day, R.; Salzet, M.; Fournier, I. MALDI-MS Direct Tissue Analysis of Proteins: Improving Signal Sensitivity Using Organic Treatments. Anal. Chem. 2006, 78, $7145-7153$.

15. Chaurand, P.; Rahman, M. A.; Hunt, T.; Mobley, J. A.; Gu, G.; Latham, J. C.; Caprioli, R. M.; Kasper, S. Monitoring Mouse Prostate Development by Profiling and Imaging Mass Spectrometry. Mol. Cell. Proteom. 2008, 7, 411-423.

16. Heeren, R. M. A.; Kaletas, B.; Taban, I. M.; MacAleese, L.; McDonnell, L. A. Quality of Surface: The Influence of Sample Preparation on MS Based Biomolecular Tissue Imaging with MALDI-MS and (ME-)SIMS. Appl. Surf. Sci. 2008, 255 1289-1297.

17. Luxembourg, S. L.; Mize, T. H.; McDonnell, L. A.; Heeren, R. M. A High-Spatial Resolution Mass Spectrometric Imaging of Peptide and Protein Distributions on a Surface. Anal. Chem. 2004, 76, 5339-5344

18. Altelaar, A. F. M.; Taban, I. M.; McDonnell, L. A.; Verhaert, P.; de Lange, R. P. J.; Adan, R. A. H.; Mooi, W. J.; Heeren, R. M. A.; Piersma,
S. R. High-Resolution MALDI Imaging Mass Spectrometry Allows Localization of Peptide Distributions at Cellular Length Scales in Pituitary Tissue Sections. Int. J. Mass Spectrom. 2007, 260, 203-211.

19. Froesch, M.; Kharchenko, A.; Luxembourg, S. L.; Verheijde, D.; Heeren, R. M. A. Imaging Mass Spectrometry Using a Delay Line Detector. Eur. J. Mass Spectrom. 2009, in press.

20. Jurchen, J. C.; Rubakhin, S. S.; Sweedler, J. V. MALDI-MS Imaging of Features Smaller than the Size of the Laser Beam. J. Am. Soc. Mass Spectrom. 2005, 6, 1654-1659.

21. Schwartz, S. A.; Reyzer, M. L.; Caprioli, R. M. Direct Tissue Analysis Using Matrix-Assisted Laser Desorption/Ionization Mass Spectrometry: Practical Aspects of Sample Preparation. J. Mass Spectrom. 2003, 38 , 699-708.

22. Lemaire, R.; Desmons, A.; Tabet, J. C.; Day, R.; Salzet, M.; Fournier, I Direct Analysis and MALDI Imaging of Formalin-Fixed, ParaffinEmbedded Tissue Sections. J. Proteome Res. 2007, 6, 1295-1305.

23. Stauber, J.; Lemaire, R.; Franck, J.; Bonnel, D.; Croix, D.; Day, R.; Wisztorski, M.; Fournier, I.; Salzet, M. MALDI Imaging of FormalinFixed Paraffin-Embedded Tissues: Application to Model Animals of Parkinson Disease for Biomarker Hunting. J. Proteome Res. 2008, 7 , 969-978.

24. Aoki, Y.; Toyama, A.; Shimada, T.; Sugita, T.; Aoki, C.; Umino, Y.; Suzuki, A.; Aoki, D.; Daigo, Y.; Nakamura, Y.; Sato, T. A. A Novel Method for Analyzing Formalin-Fixed Paraffin Embedded (FFPE) Tissue Sections by Mass Spectrometry Imaging. Proc. Jpn. Acad. B Phys. Biol. Sci. 2007, 83, 205-214.

25. McLean, J. A.; Stumpo, K. A.; Russell, D. H. Size-Selected (2-10 nm) Gold Nanoparticles for Matrix Assisted Laser Desorption Ionization of Peptides. J. Am. Chem. Soc. 2005, 127, 5304-5305.

26. Taira, S.; Sugiura, Y.; Moritake, S.; Shimma, S.; Ichiyanagi, Y.; Setou, M. Nanoparticle-Assisted Laser Desorption/Ionization Based Mass Imaging with Cellular Resolution. Anal. Chem. 2008, 80, 4761-4766.

27. Edirisinghe, P. D.; Moore, J. F.; Calaway, W F.; Veryovkin, I. V.; Pellin M. J.; Hanley, L. Vacuum Ultraviolet Post-Ionization of Aromatic Groups Covalently Bound to Peptides. Anal. Chem. 2006, 78, 5876-5883.

28. Hanley, L.; Kornienko, O.; Ada, E. T.; Fuoco, E.; Trevor, J. L. Surface Mass Spectrometry of Molecular Species. J. Mass Spectrom. 1999, 34, 705-723.

29. Stauber, J.; Lemaire, R.; Franck, J.; Bonnel, D.; Croix, D.; Day, R.; Wisztorski, M.; Fournier, I.; Salzet, M. MALDI Imaging of FormalinFixed Paraffin-Embedded Tissues: Application to Model Animals of Parkinson Disease for Biomarker Hunting. J. Proteome Res. 2008, 7, 969-978.

30. Groseclose, M. R.; Andersson, M.; Hardesty, W. M.; Caprioli, R. M. Identification of Proteins Directly from Tissue: In Situ Tryptic Digestions Coupled with Imaging Mass Spectrometry. J. Mass Spectrom. 2007, $42,254-262$.

31. Rohner, T. C.; Staab, D.; Stoeckli, M. MALDI Mass Spectrometric Imaging of Biological Tissue Sections. Mech. Aging Dev. 2005, 126, 177-185.

32. Taban, I. M.; Altelaar, A. F. M.; van der Burgt, Y. E. M.; McDonnell, L. A.; Baykut, G.; Heeren, R. M. A. Imaging of Peptides in the Rat Brain Using MALDI-FTICR Mass Spectrometry. J. Am. Soc. Mass Spectrom. 2007, 18, 145-151.

33. Garrett, T. J.; Prieto-Conaway, M. C.; Kovtoun, V.; Bui, H.; Izgarian, N.; Stafford, G.; Yost, R. A. Imaging of Small Molecules in Tissue Sections with a New Intermediate-Pressure MALDI Linear Ion Trap Mass Spectrometer. Int. J. Mass Spectrom. 2007, 260, 166-176.

34. Shimma, S.; Sugiura, Y.; Hayasaka, T.; Zaima, N.; Matsumoto, M.; Setou, M. Mass Imaging and Identification of Biomolecules with MALDI-QIT-TOF-Based System. Anal. Chem. 2008, 80, 878-885.

35. McLean, J. A.; Ruotolo, B. T.; Gillig, K. J.; Russell, D. H. Ion MobilityMass Spectrometry: A New Paradigm for Proteomics. Int. J. Mass Spectrom. 2005, 240, 301-315.

36. Verbeck, G.; Ruotolo, B.; Sawyer, H.; Gillig, K.; Russell, D. A Fundamental Introduction to Ion Mobility Mass Spectrometry Applied to the Analysis of Biomolecules. J. Biomol. Tech. 2002, 13, 56-61.

37. Clemmer, D. E.; Jarrold, M. F. Ion Mobility Measurements and Their Applications to Clusters and Biomolecules. J. Mass Spectrom. 1997, 32 577-592.

38. Vonhelden, G.; Wyttenbach, T.; Bowers, M. T. Inclusion of a MALDI Ion-Source in the Ion Chromatography Technique-Conformational Information on Polymer and Biomolecular Ions. Int. J. Mass Spectrom. Ion Processes 1995, 146, 349-364.

39. Ruotolo, B. T.; McLean, J. A.; Gillig, K. J.; Russell, D. H. Peak Capacity of Ion Mobility Mass Spectrometry: The Utility of Varying Drift Gas Polarizability for the Separation of Tryptic Peptides. J. Mass Spectrom. 2004, 39, 361-367.

40. Ruotolo, B. T.; Verbeck, G. F.; Thomson, L. M.; Woods, A. S.; Gillig, K. J.; Russell, D. H. Distinguishing Between Phosphorylated and Nonphosphorylated Peptides with Ion Mobility-Mass Spectrometry. J. Proteome Res. 2002, 1, 303-306.

41. Hatsis, P.; Kapron, J. T. A Review on the Application of High-Field Asymmetric Waveform Ion Mobility Spectrometry (FAIMS) in Drug Discovery. Rapid Commun. Mass Spectrom. 2008, 22, 735-738.

42. Williams, J. P.; Scrivens, J. H. Coupling Desorption Electrospray Ionization and Neutral Desorption/Extractive Electrospray Ionization with a Traveling-Wave Based Ion Mobility Mass Spectrometer for the Analysis of Drugs. Rapid Commun. Mass Spectrom. 2008, 22, 187-196. 
43. J. Stauber, B. K. K.; van der Wiel, I. M.; Snel, M. F.; Claude, E.; Heeren, R. M. A. Ion Mobility Imaging Mass Spectrometry: A New Tool for In Situ Identification. Proceedings of the 56th ASMS conference; Denver, CO, June, 2008.

44. Jackson, S. N.; Ugarov, M.; Egan, T.; Post, J. D.; Langlais, D.; Schultz, J. A.; Woods, A. S. MALDI-Ion Mobility-TOF-MS Imaging of Lipids in Rat Brain Tissue. J. Mass Spectrom. 2007, 42, 1093-1098.

45. Tempez, A.; Ugarov, M.; Egan, T.; Schultz, J. A.; Novikov, A.; DellaNegra, S.; Lebeyec, Y.; Pautrat, M.; Caroff, M.; Smentkowski, V. S.;
Wang, H.-Y. J.; Jackson, S. N.; Woods, A. S. Matrix Implanted Laser Desorption Ionization (MILDI) Combined with Ion Mobility-Mass Spectrometry for Biosurface Analysis. J. Proteome Res. 2005, 4, 540-545. 46. Woods, A. S.; Ugarov, M.; Egan, T.; Koomen, J.; Gillig, K. J.; Fuhrer, K.; Gonin, M.; Schultz, J. A. Lipid/Peptide/Nucleotide Separation with MALDI-Ion Mobility-TOF MS. Anal. Chem. 2004, 76, 2187-2195.

47. Overton, W. R. Catalano, E.; McCoy, J. P. Method to Make ParaffinEmbedded Breast and Lymph Tissue Mimic Fresh Tissue in DNA Analysis. Cytometry 1996, 26, 166-171. 